\title{
Shubnikov-de Haas oscillations of two-dimensional electron gas in an InAsN/InGaAs single quantum well
}

\author{
D R Hang ${ }^{1}$, C F Huang ${ }^{1}$, W K Hung ${ }^{1}$, Y H Chang ${ }^{1}$, J C Chen ${ }^{1}$, \\ H C Yang ${ }^{1}$, Y F Chen ${ }^{1}$, D K Shih', T Y Chu' and H H Lin ${ }^{2}$ \\ ${ }^{1}$ Department of Physics, National Taiwan University, 106 Taipei, Taiwan, Republic of China \\ ${ }^{2}$ Department of Electrical Engineering, National Taiwan University, 106 Taipei, Taiwan, \\ Republic of China
}

Received 2 May 2002, in final form 4 July 2002

Published 9 August 2002

Online at stacks.iop.org/SST/17/999

\begin{abstract}
We present the first investigation of Shubnikov-de Haas ( $\mathrm{SdH}$ ) oscillations of two-dimensional electron gas formed in an InAsN/InGaAs single quantum well $(\mathrm{QW})$ grown on an InP substrate using gas source molecular beam epitaxy and a radio-frequency (rf) plasma nitrogen source. The photoluminescence (PL) peak energy of the InAsN/InGaAs QW decreases compared with that of InAs/InGaAs QW. This agrees with the bowing effect due to the incorporation of nitrogen atoms. The nitrogen content can be estimated to be $0.4 \%$ using the PL peak positions as well as X-ray diffraction. From the $\mathrm{SdH}$ oscillations, the carrier concentration is $2.85 \times$ $10^{11} \mathrm{~cm}^{-2}$ and the effective mass is $0.1 \pm 0.01 m_{0}$. The enhancement of the effective mass is mainly due to the incorporation of the nitrogen atoms in the InAs lattice, which is consistent with a recent study on InAsN bulk alloys. The large increase of the effective mass cannot be explained by the simple band anticrossing model. In addition, we observe a temperatureindependent magnetoresistivity at a critical magnetic field. Our analysis supports the fact that the value of the critical exponent in the quantum Hall effect is not universal.
\end{abstract}

\section{Introduction}

In recent years, III-V alloys containing nitrogen have received much attention because of their potential application for infrared devices and their interesting physical properties [1-3]. In contrast to conventional III-V alloys, such as GaInP, in which the electronic properties vary smoothly with composition, the incorporation of even a small fraction of nitrogen into the host III-V crystal results in drastic modifications of the electronic properties of the alloys [4-7]. The most renowned peculiar behaviour is the significant bandgap reduction, usually called 'giant bowing' [4]. For example, a $0.4 \mathrm{eV}$ shrinkage of the fundamental bandgap has been reported in $\mathrm{GaAs}_{1-x} \mathrm{~N}_{x}$ with $x=4 \%$ [7]. This anomaly in the III-N-V alloys has motivated numerous theoretical efforts to understand the observed experimental results [8-12]. Yet the actual underlying mechanism is still being debated. In the band anticrossing (BAC) model, the resonant $\mathrm{N}$ level repulses the extended conduction-band states and splits the conduction band into two nonparabolic subbands [8]. Within a similar framework, theoretical studies based on the pseudopotential supercell calculations suggest that the mixing between the $\Gamma$ and $\mathrm{L}$ states, as a consequence of a complex perturbation of the lowest conduction-band states by nitrogen substitution, is the microscopic physical origin [11]. On the other hand, Zhang et al [12] have proposed that the formation of an impurity band and the quantum confinement of an electron to the impurity band account for the abnormal phenomena. Hence, this issue still needs further theoretical and experimental investigations.

For optoelectronic devices working in the mid-infrared 2-5 $\mu \mathrm{m}$ wavelengths, the InAs-based heterostructure has been adopted because of its narrow bandgap $(0.36 \mathrm{eV}$ at room temperature). Wang et al [13] have demonstrated a high-quality $2.2 \mu \mathrm{m} \mathrm{InAs/InGaAs/InP} \mathrm{highly} \mathrm{strained}$ multiple quantum well (QW) laser grown by gas source molecular beam epitaxy. Recently, it has been shown that 
a better heterointerface can be achieved by replacing InAs with InAsN alloys since nitrogen atoms are smaller than arsenic [14]. In addition, the bandgap energy of InAsN alloys can further be reduced because of the giant bowing and the fact that the larger band discontinuity results in a better carrier confinement. Therefore, research into InAsN improves optoelectronic device qualities, pushes laser emission to longer wavelengths and broadens our knowledge of III-N-V alloys. However, previously published reports have concentrated mainly on the optical properties [14-17], and as yet there has been no transport study of InAsN-based heterostructures. In order to be probed by transport experiments, the sample quality must be improved to a certain degree, which requires excellent epitaxial knowledge. In this paper, we report on the first Shubnikov-de Haas $(\mathrm{SdH})$ oscillations of two-dimensional electron gas (2DEG) formed in an InAsN/InGaAs single QW with a nitrogen composition of $0.4 \%$. Using the temperaturedependent $\mathrm{SdH}$ oscillations, the effective mass is found to be $0.1 \pm 0.01 m_{0}$. The enhancement of the effective mass is mainly due to the band structure modification induced by nitrogen. However, the simple BAC model may be unsuitable to account for the experimental observation, which is in contrast to the case of InGaNAs. This result is consistent with a recent study on InAsN bulk alloys.

\section{Experimental details}

The samples used in this study were grown on semi-insulating InP (100) substrates by a VG V-80H gas source molecular beam epitaxy system. Elemental In and Ga sources and a thermally cracked $\mathrm{AsH}_{3}$ source were used to produce molecular beams. The active $\mathrm{N}$ species were generated from an EPI UNI-bulb radio-frequency (rf) plasma source. The rf power for the growths was kept at $180 \mathrm{~W}$. The QW structures were grown on InP substrates and consisted of a $0.3 \mu \mathrm{m}$ thick $\mathrm{In}_{0.53} \mathrm{Ga}_{0.47}$ As layer which was lattice matched to InP, a $3 \mathrm{~nm}$ thick $\operatorname{InAs}(\mathrm{N})$ well and a $40 \mathrm{~nm}$ thick $\mathrm{In}_{0.53} \mathrm{Ga}_{0.47} \mathrm{As}$ barrier. The growth temperature was $400{ }^{\circ} \mathrm{C}$. More details of the growth conditions can be found elsewhere [14]. The $\operatorname{InAs}(\mathrm{N})$ QWs were first examined by photoluminescence (PL) measurements. The PL measurements were carried out in a closed cycle helium cryostat. The $488 \mathrm{~nm}$ line of an argon laser was used for the excitation source. The signal was detected by a liquid-nitrogen cooled $\mathrm{InSb}$ photodiode with a Hamamatsu P3357-02 pre-amplifier. Figure 1 shows the 10 K PL spectra of the InAs(N) QW structures. As can be seen, the PL peak energy of the InAsN QW decreases because of the incorporation of nitrogen. This result confirms the giant bowing effect. From the amount of the PL peak position shift and from x-ray diffraction, the nitrogen composition is estimated to be $0.4 \%$ [18].

For the transport measurement, ohmic contacts were formed by depositing indium drops at the corners of the samples, and by annealing the contacts at $400{ }^{\circ} \mathrm{C}$ for $10 \mathrm{~s}$. The samples were immersed in liquid helium. The properties of the 2DEG formed in the InAsN/InGaAs QW were investigated by magnetotransport measurements at low temperatures and at magnetic fields of up to $7 \mathrm{~T}$ in a ${ }^{3} \mathrm{He}$ refrigerator. The temperature could be cooled down to as low as $0.3 \mathrm{~K}$ and could be controlled from $0.3 \mathrm{~K}$ to $1.2 \mathrm{~K}$ by carefully adjusting

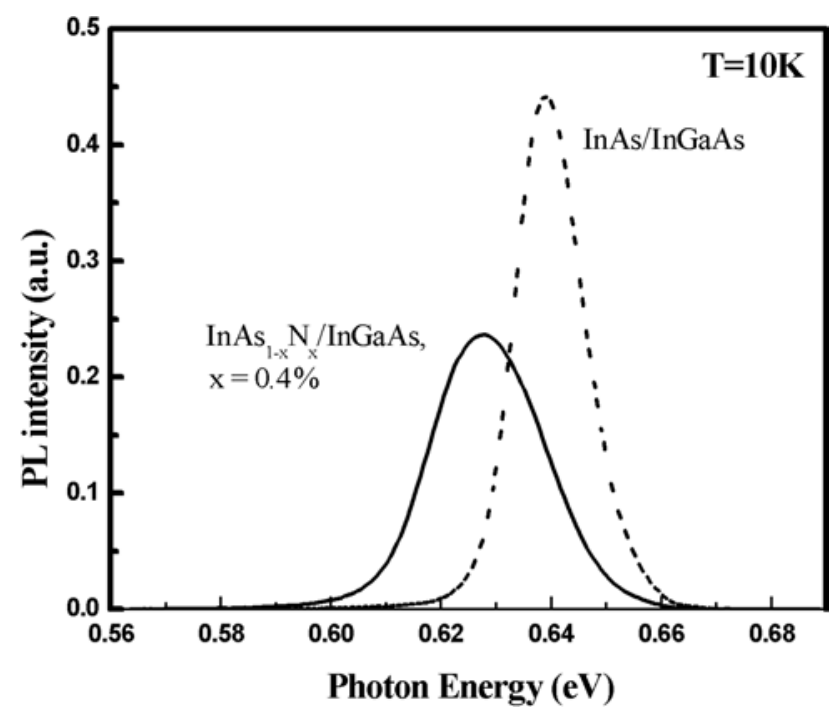

Figure 1. PL spectra of $\operatorname{InAs}(\mathrm{N}) \mathrm{QWs}$ taken at a temperature of $10 \mathrm{~K}$.

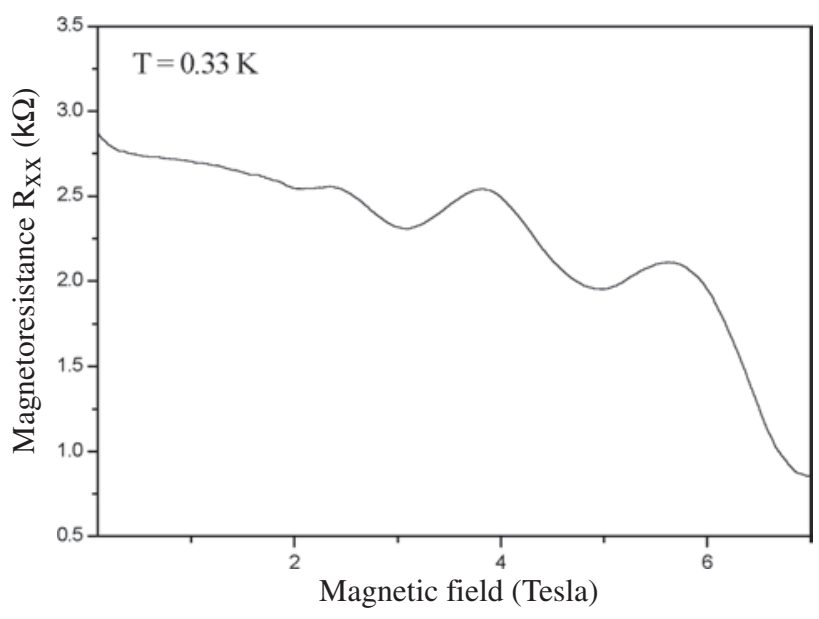

Figure 2. Typical SdH oscillations of the InAsN QW sample at a temperature of $0.33 \mathrm{~K}$.

the heater. It was also possible to reach higher temperatures of up to $2.1 \mathrm{~K}$ by adjusting the ${ }^{4} \mathrm{He}$ absorption rate. The data were taken by conventional lock-in techniques. During the measurements, the current was monitored and kept small to avoid the electron-heating effect.

\section{Measurements of $\mathrm{SdH}$ oscillations}

The $\mathrm{SdH}$ measurement has been very well received as a tool for studying the properties of 2D carriers, such as the effective mass, the carrier concentration and the scattering time. Figure 2 shows the SdH oscillations of the InAsN/InGaAs QW taken at $0.33 \mathrm{~K}$. There is only one series of oscillations and the $\mathrm{SdH}$ oscillations can be resolved for magnetic fields higher than $2 \mathrm{~T}$. From the onset of the $\mathrm{SdH}$ oscillations, the mobility near the liquid-helium temperature is roughly estimated to be $5000 \mathrm{~cm}^{2} \mathrm{~V}^{-1} \mathrm{~s}^{-1}$, which suggests good crystalline quality. The $2 \mathrm{D}$ character has been further confirmed by rotating the sample orientation against the magnetic field. 


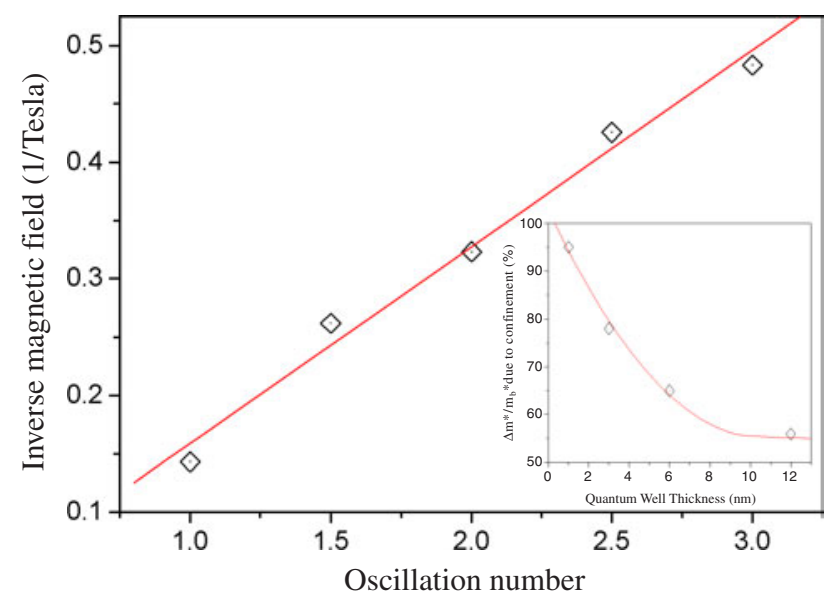

Figure 3. The inverse magnetic field of the $\mathrm{SdH}$ minimum as a function of the successive oscillation number (open diamonds). The solid line shows the fit to equation (2). The inset shows the percentile effective mass increase due to size quantization as a function of well thickness. The solid line is drawn as a guide to the eye. $m_{b}^{*}$ denotes the bulk mass and $\Delta m^{*}$ represents the increase of the bulk mass.

The oscillatory part $\Delta \rho_{x x}$ of the $\mathrm{SdH}$ oscillations at a temperature $T$ can be expressed as [19]

$$
\Delta \rho_{x x} \approx 4 \rho_{0} \frac{Z}{\sinh (Z)} \exp \left(-\pi / \omega_{\mathrm{c}} \tau\right)
$$

where $\rho_{0}$ is the zero-field resistivity, $\tau$ is the quantum lifetime, $Z=2 \pi^{2} k_{\mathrm{B}} T / \hbar \omega_{\mathrm{c}}, \hbar$ is the reduced Planck constant, $k_{\mathrm{B}}$ is the Boltzmann constant, the cyclotron frequency $\omega_{\mathrm{c}}=\mathrm{eB} / \mathrm{m}^{*}$, $e$ is the electron charge, $B$ is the magnetic field, and $m^{*}$ is the electron effective mass.

The inverse magnetic fields of the minima of the $\mathrm{SdH}$ oscillations as a function of the successive oscillation numbers are plotted as open diamonds in figure 3. The data can be described by the simple linear equation [20]

$$
\frac{1}{B_{N}}=N \frac{e}{\pi \hbar n}+C
$$

where $B_{N}$ represent magnetic fields at successive oscillation minima, $N$ is an integer, $C$ is a constant, and $n$ is the carrier concentration. The choice of the oscillation number is arbitrary. The solid line in figure 3 shows the fit to equation (2). The slope of the line corresponds to the carrier density $2.85 \times 10^{11} \mathrm{~cm}^{-2}$.

The oscillation amplitudes can be found to increase with the decrease of temperature and the effective mass can be obtained by fitting temperature-dependent amplitudes of the $\mathrm{SdH}$ oscillations to the form following from equation (1)

$$
\frac{A_{1}\left(B, T_{1}\right)}{A_{2}\left(B, T_{2}\right)}=\frac{Z_{1} \sinh Z_{2}}{Z_{2} \sinh Z_{1}} \text {. }
$$

Here, $A_{i}$ is the amplitude of the oscillation and $B$ is the magnetic field value at which the amplitudes are taken. The effective mass is determined by obtaining the solution of equation (3) as $0.1 \pm 0.01 m_{0}$. It is also possible to fit the amplitudes by the conventional plot of $\ln (A / T)$ versus $T$, as long as $Z \gg 1$ [21].

The experimental effective mass value obtained here is evidently much higher than the nitrogen-free value, $m^{*}(\mathrm{InAs}) \sim 0.026 m_{0}[22]$. When electrons are confined in an InAsN QW, the electron effective mass can be enhanced due to the reduced dimension. To assess the extent of such a modification of the effective mass, we have quantitatively estimated the mass enhancement due to size quantization by a self-consistent calculation based on a finite-depth single QW model. We show the percentile increase of the effective mass as a function of the QW thickness in the inset of figure 3. For the $3 \mathrm{~nm}$ wide QW that we have studied, the increase of the effective mass is about $77 \%$. It is clear that the sizequantization effect alone cannot account for the large effective mass enhancement that we have observed. Besides, the studied sample is a narrow bandgap semiconductor, and the nonparabolicity effect may contribute to the enhancement of the effective mass. By taking account of the $k \cdot p$ approximation, the effective mass at the Fermi level can be expressed as

$$
m^{*}\left(E_{F}\right)=m_{0}^{*}\left(1+2 E_{F} / E_{g}\right),
$$

where $m^{*}\left(E_{F}\right)$ is the effective mass at the Fermi level, $m_{0}^{*}$ is the effective mass at the band edge and $E_{g}$ is the bandgap. The bandgap energy of InAsN is found to be $462 \mathrm{meV}$ at a low temperature from our PL study [18]. The Fermi energy can be determined by $E_{F}=\pi \hbar n / m^{*}$, where the carrier concentration $n$ and the effective mass $m^{*}$ can be obtained from the above measurements. The calculated Fermi energy is $6.75 \mathrm{meV}$. Hence, we have found that the enhancement of the effective mass due to the nonparabolic effect is only $2.9 \%$, which is substantially less than the observed result. As a consequence, the mass enhancement is mainly due to the incorporation of the nitrogen into the host lattice. This result is in agreement with previous theoretical studies proposing that the strong interaction or admixture of the conductionband minimum with upper states gives rise to an increase of the electron effective mass [8-11]. In the simple BAC model, the resonant $N$ states above the conduction-band minimum interact with the extended states of the conduction band, splitting the conduction band into two nonparabolic subbands. The lower flattened subband brings about the increase of the electron effective mass. In addition, one further notes that within this theoretical framework, the electron effective mass cannot exceed $2 m_{M}$ if the nitrogen-induced level is higher than the conduction-band minimum [10]. Here, $m_{M}$ denotes the nitrogen-free electron effective mass in the host lattice. However, our measured value of the effective mass clearly exceeds $2 m_{M}$. This result is consistent with a recent study of bulk InAs ${ }_{1-x} \mathrm{~N}_{x}$ by means of infrared reflectivity, which found that the measured effective masses either approach $2 m_{M}$ or are extremely larger than $2 m_{M}{ }^{17}$. Consequently, the simple BAC model seems to be inadequate for InAsN though it works well for InGaNAs. Another refined form of the BAC model suggests that the conduction band edge is a mixing state caused by the strong perturbation introduced by smaller nitrogen atoms on arsenic sites [11]. The mixing state consists of components of the $\Gamma, \mathrm{L}$ and $\mathrm{X}$ states and the $\Gamma$ character decreases as the nitrogen composition increases. Since the $\mathrm{L}$ electrons have much heavier mass than the $\Gamma$, the electron effective mass can possibly exceed $2 m_{M}$. The mixing state model therefore supplies a plausible explanation. Nevertheless, the exact mechanism of the band structure modification still calls for further investigation. 


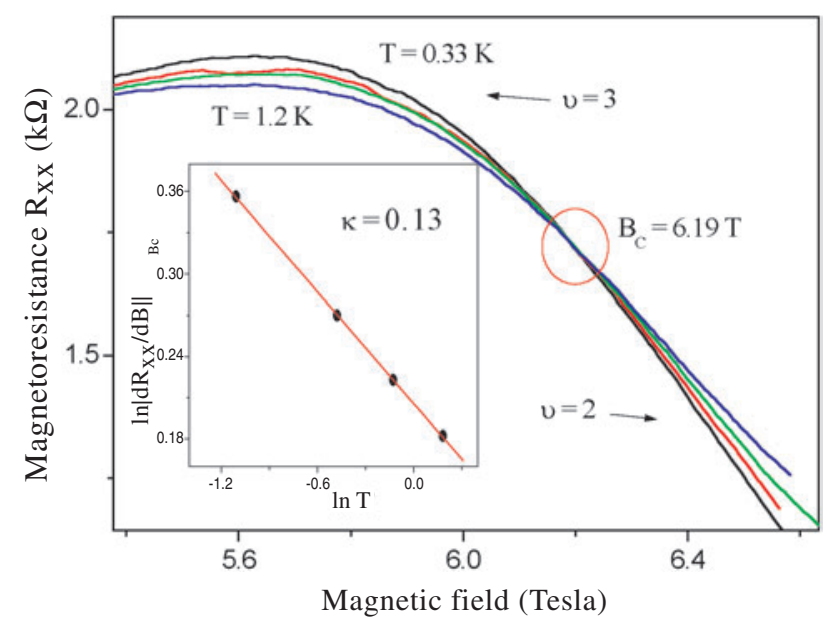

Figure 4. The magnetoresistance curves near the temperature-independent point. For $B<B_{\mathrm{c}}$, the curves are obtained at temperatures of $0.33,0.62,0.88$ and $1.2 \mathrm{~K}$ (from top to bottom). The inset shows the plot of $\ln \left|\mathrm{d} R_{x x} / \mathrm{d} B\right|_{B_{\mathrm{c}}}$ versus $\ln T$. A good linearity is shown and the scaling exponent is obtained from the slope to be $\kappa=0.13 \pm 0.01$.

Finally, we briefly remark on observed interesting phenomena in the high field part of the magnetoresistance. As can be seen in figure 4 , the longitudinal sheet resistance $R_{x x}$ (also $\rho_{x x}$, since $R_{x x}$ differs from $\rho_{x x}$ by a geometric factor) appears to be temperature independent at $B=6.19 \mathrm{~T}$. For convenience, we denote this magnetic field by $B_{\mathrm{c}}$. We also observed a Hall plateau of $n=2$ in the sample even though the sample suffers from few parallel conductions. Between $v=2$ and 3 quantum Hall states, as shown in figure 4, the temperature-independent point can be the critical point separating these two quantum Hall states [23, 24]. It is known that at low temperatures both longitudinal conductivity $\sigma_{x x}$ and transverse conductivity $\sigma_{x y}$ are functions of $\left(B-B_{\mathrm{c}}\right) / T^{\kappa}$ near such a point. Hence

$$
\rho_{x x}=\sigma_{x x} /\left(\sigma_{x x}^{2}+\sigma_{x y}^{2}\right)=f\left(\left(B-B_{\mathrm{c}}\right) / T^{\kappa}\right),
$$

where $\kappa$ is the critical exponent and $f$ is a universal scaling function [25-27]. Wei et al [28] found that $\kappa=0.42 \pm 0.04$ as a universal value. However, Koch et al [29] found that $\kappa$ is sample dependent, and is even dependent on the Landau level within the same sample. From equation (5), by applying Taylor expansion around $B=B_{\mathrm{c}}$ and by keeping the first term, we obtain $\left|\mathrm{d} \rho_{x x} / \mathrm{d} B\right|_{B=B \mathrm{c}} \sim T^{-\kappa}$. A scaling behaviour can be checked by plotting $\ln \left|\mathrm{d} R_{x x} / \mathrm{d} B\right|_{B=B_{c}}$ versus $\ln T$, which is shown in the inset of figure 4. A good linearity is shown and the slope yields $\kappa \sim 0.13 \pm 0.01$. This result is consistent with previous reports that $\kappa$ is not universal [27, 29].

\section{Conclusions}

In summary, we present the first investigation of $\mathrm{SdH}$ oscillations of 2DEG formed in an InAsN/InGaAs single QW. The nitrogen composition is estimated to be $0.4 \%$ using the PL peak positions as well as x-ray diffraction. The sheet carrier concentration determined from $\mathrm{SdH}$ oscillations is $2.85 \times 10^{11} \mathrm{~cm}^{-2}$ and the effective mass evaluated from the temperature-dependent $\mathrm{SdH}$ amplitudes is $0.1 \pm 0.01 m_{0}$. The enhancement of the effective mass is due to the band structure modification induced by nitrogen. The large increase of the effective mass cannot be explained within the framework of the simple BAC model. Our result is useful for reexamining the validity of assumptions in different theoretical approaches and for the optimization of devices based on InAsN/InGaAs QWs. There needs to be further studies on the complex perturbation induced by the nitrogen state. A temperatureindependent $R_{x x}$ (or $\rho_{x x}$ ) point at $B=6.19 \mathrm{~T}$ is observed which is the critical point separating $v=2$ and 3 quantum Hall states. Near the critical magnetic field, the magnetoresistivity follows a universal scaling function with the critical exponent $\kappa \sim 0.13 \pm 0.01$. This supports the fact that the value of the critical exponent is not universal.

\section{Acknowledgments}

This work was partly supported by the National Science Council and the Ministry of Education of the Republic of China.

\section{References}

[1] Kondow M, Uomi K, Kitatani T, Watahiki S and Yazawa Y 1996 J. Cryst. Growth 164175

[2] Kinsey G S, Gotthold D W, Holmes A L Jr, Streetman B G and Campbell J C 2000 Appl. Phys. Lett. 762824

[3] Yu K M, Walukiewicz W, Wu J, Beeman J W, Ager J W III, Haller E E, Shan W, Xin H P and Tu C W 2001 Appl. Phys. Lett. 781077

[4] Geisz J F, Friedman D J, Olson J M, Kurtz S R and Keyes B M 1998 J. Cryst. Growth 195401

[5] Weyers M, Sato M and Ando H 1992 Japan.J. Appl. Phys. 31 L853

[6] Suemune I, Uesugi K and Walukiewicz W 2000 Appl. Phys. Lett. 773021

[7] Uesugi K, Marooka N and Suemune I 1999 Appl. Phys. Lett. 741254

[8] Shan W, Walukiewicz W, Ager J W III, Haller E E, Geisz J F, Friedman D J, Olson J M and Kurtz S R 1999 Phys. Rev. Lett. 821221

[9] Lindsay A and O'Reilly E P 1999 Solid State Commun. 112 443

[10] Skierbiszewski C et al 2000 Appl. Phys. Lett. 762409

[11] Mattila T, Wei S H and Zunger A 1999 Phys. Rev. B 60 R11245

[12] Zhang Y, Mascarenhas A, Xin H P and Tu C W 2000 Phys. Rev. B 617479

[13] Wang J S, Lin H H and Song L W 1998 IEEE J. Quantum Electron. 341959

[14] Wang J S, Lin H H, Song L W and Chen G R 2001 J. Vac. Sci. Technol. B 19202

[15] Wang J S and Lin H H 1999 J. Vac. Sci. Technol. B 171997

[16] Fan J C, Hung W K, Chen Y F, Wang J S and Lin H H 2000 Phys. Rev. B 6210990

[17] Hung W K, Cho K S, Chern M Y, Chen Y F, Shih D K, Lin H H, Lu C C and Yang T R 2002 Appl. Phys. Lett. 80 796

[18] Chu T Y, Lin H H and Shih D K 2001 Electron Devices and Material Symposia (Kaohsiung, Taiwan, ROC) pp 126-9

[19] Wong L W, Cai S J, Li R, Wang K, Jiang H W and Chen M 1998 Appl. Phys. Lett. 731391 
[20] Merino J and McKenzie R H 2000 Phys. Rev. B 62 2416

[21] Saxler A et al 2000 J. Appl. Phys. 87369

[22] Vurgaftman I, Meyer J R and Ram-Mohan L R $2001 \mathrm{~J}$. Appl. Phys. 895815

[23] Pruisken A M M 1988 Phys. Rev. Lett. 611297

[24] Dolan B P 2000 Phys. Rev. B 6210278

[25] Wang T, Clark K P, Spencer G F, Mack A M and Kirk W P 1994 Phys. Rev. Lett. 72709
[26] Lee C H, Chang Y H, Suen Y W and Lin H H 1998 Phys. Rev. B 5810629

[27] Huang C F, Chang Y H, Lee C H, Chou H T, Yeh H D, Liang C-T, Chen Y F, Lin H H, Cheng H H and Hwang G J 2001 Phys. Rev. B 65045303

[28] Wei H P, Tsui D C, Paalanen M A and Pruisken A M M 1988 Phys. Rev. Lett. 611294

[29] Koch S, Haug R J, von Klitzing K and Ploog K 1991 Phys. Rev. B 436828 